[0212-7199 (2004) 21: 4; pp 175-178] ANALES DE MEDICINA INTERNA Copyright $(C) 2004$ ARAN EDICIONES, S.L.

AN. MED. INTERNA (Madrid) Vol. 21, N. $^{\circ} 4$, pp. $175-178,2004$

\title{
Linfoma primario de cavidades asociado a infección por VIH: características clínico-biológicas en tres pacientes diagnosticados en un mismo centro
}

\author{
M. J. RODRÍGUEZ SALAZAR, J. M. RAYA SÁNCHEZ, R. RODRÍGUEZ SÁNCHEZ, \\ M. M. ALONSO SOCAS ${ }^{1}$, M. L. BRITO BARROSO, L. HERNÁNDEZ NIETO \\ Servicios de Hematología y ${ }^{\prime}$ Medicina Interna. Hospital Universitario de Canarias. La \\ Laguna. Santa Cruz de Tenerife
}

HIV-ASSOCIATED PRIMARY BODY-CAVITY-BASED LYMPHOMA: CLINICO-BIOLOGIC FEATURES IN THREE PATIENTS DIAGNOSED AT THE SAME INSTITUTION

\begin{abstract}
RESUMEN
El linfoma primario de cavidades (LPC) constituye un variedad de linfoma no Hodgkin individualizada por la clasificación OMS, que se desarrolla principalmente en pacientes varones con infección por HIV, más frecuentemente homosexuales y en estadios avanzados de la enfermedad (recuento total de linfocitos CD4+ inferior a 100-200/ $\mu \mathrm{L}$ ), aunque en ocasiones pueden aparecer en otras circunstancias asociadas a estados de inmunodepresión (como puede ser en el postrasplante de órganos sólidos) e incluso, de forma muy ocasional, en pacientes inmunocompetentes. Desde un punto de vista patogenético se ha relacionado con el virus herpes asociado a sarcoma de Kaposi (también denominado virus herpes tipo 8) y al propio antecedente clínico de sarcoma de Kaposi. La relativa rareza de esta enfermedad, la falta de casuísticas amplias que logren caracterizarla mejor y su pronóstico tan desfavorable, obligan a profundizar en un mejor conocimiento de la misma. Presentamos los hallazgos clínico-biológicos de tres pacientes diagnosticados de LPC pleural en nuestro centro en los últimos dos años.
\end{abstract}

PALABRAS CLAVE: Virus de la inmunodeficiencia humana, VIH. Linfoma primario de cavidades.

\begin{abstract}
Primary effusion lymphoma (PEL) is a recently individualized form of non-Hodgkin lymphoma (WHO classification) that mainly develops in HIV infected males, more frequently in homosexuals and advanced sta-

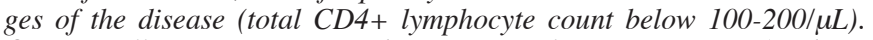
Occasionally, it appears in others immunodepressive states (such as solid organs postransplant period) and even, although very rarelly, in immunocompetents patients. From a pathogenetic point of view, PEL has been related to Kaposi's sarcoma-associated herpes virus (also named human herpesvirus 8) and to the clinical antecedent of Kaposi's sarcoma. Relative unfrequency of this disease, the absence of wide casuistics allowing a better characterization, and its unfavorable outcome, support the need of a deeper knowledge. We present here the clinical-biological findings of three patients that were diagnosed of pleural PEL in our institution in the last two years.
\end{abstract}

KEY WORDS: Human immunodeficiency virus, HIV. Primary effusion lymphoma.

M. J. Rodríguez Salazar, J. M. Raya Sánchez, R. Rodríguez Sánchez, Ma del M. Alonso Socas 1, M. L. Brito Barroso, L. Hernández Nieto. Linfoma primario de cavidades asociado a infección por VIH: características clínico-biológicas en tres pacientes diagnosticados en un mismo centro. An Med Interna (Madrid) 2004; 21: 175-178.

\section{INTRODUCCIÓN}

La predisposición de los pacientes seropositivos para el VIH a desarrollar neoplasias y en concreto linfomas, es un hecho conocido. En Europa, la prevalencia de linfomas no Hodgkin en pacientes con sida experimentó un incremento del $3,6 \%$ al 5,4\% entre 1994 y 2000 (1). La presencia de un derrame pleural en un paciente positivo para el virus de la inmunodeficiencia humana (VIH), si bien en absoluto específico, nos debe hacer pensar en la posibilidad de un linfoma primario de cavidades (LPC) (2). Aunque las clasificaciones más recientes de los linfomas no Hodgkin consideran el LPC como una entidad específica basándose para ello principalmente en estudios moleculares e inmunofenotípicos, lo cierto es que no existen casuísticas amplias recogidas en la bibliografía y que se hace necesaria una mejor caracterización del proceso. Se piensa que desde un punto de vista etiopatogénico, el virus herpes asociado a sarcoma de Kaposi (también denominado virus herpes tipo 8 o KSHV) puede jugar un papel importante; menos clara está su relación con el virus de Epstein-Barr (VEB) y con el antecedente clínico de sarcoma de Kaposi (SK). Presentamos los hallazgos clínico-biológicos de tres pacientes, diagnosticados de LPC pleural en nuestro centro, en los dos últimos años.

\section{CASOS APORTADOS}

En los tres casos se trataba de sujetos varones con infección por VIH, en estadio avanzado de la enfermedad (B3 o C3). En la

Trabajo aceptado: 1 de octubre de 2003

Correspondencia: José María Raya Sánchez. Apartado de Correos 172. 38080 Santa Cruz de Tenerife. 
Tabla I se presentan las características clínico-biológicas de estos pacientes.

Quisieramos destacar el hecho de que las adenopatías biopsiadas en los casos 1 y 2 no fueron específicas de linfoma, sino de origen tuberculoso o afectadas por sarcoma de Kaposi, respectivamente. La especificidad de estas biopsias habría anulado el carácter primario del linfoma de cavidades. Por otra parte, el antecedente clínico de sarcoma de Kaposi sólo estaba presente en uno de los tres pacientes. Una mayor o menor carga viral no parece guardar relación con el proceso, pero sí una cifra de linfocitos CD4+ inferior a $200 / \mu \mathrm{L}$.

\section{DISCUSIÓN}

La infección por el virus VIH predispone al desarrollo de neoplasias, principalmente sarcoma de Kaposi, linfomas no Hodgkin, linfoma primario del SNC y carcinoma cervical invasivo, y más recientemente se ha relacionado con el linfoma primario de cavidades (LPC). En la recientemente publicada clasificación de tumores y tejidos hematopoyéticos y linfoides (3), parte de la más amplia Clasificación de Tumores de la Organización Mundial de la Salud (OMS), el LPC constituye una entidad clinico-patológica específica, diferenciada del resto de linfomas. Esta enfermedad se asocia con frecuencia a situaciones de inmunodepresión (gene- ralmente infección por VIH, pero también, por ejemplo, en pacientes trasplantados de órganos sólidos) (4,5), habiéndose descrito ocasionalmente en pacientes HIV negativos (6) y sujetos inmunocompetentes. En los pacientes con infección por VIH, el LPC suele aparecer en sujetos varones homosexuales en estadios avanzados de la enfermedad, generalmente con recuentos de células CD4 inferiores a $200 / \mu \mathrm{L}$, y con mayor frecuencia aún, inferiores a $100 / \mu \mathrm{L}$. Desde un punto de vista etiopatogénico, se ha relacionado con el virus herpes asociado a sarcoma de Kaposi, KSHV (también denominado virus herpes tipo 8 o HHV-8) (7-10). Las células malignas contienen, con mucha frecuencia, material genómico del mencionado virus KSHV / HHV-8, y en algunos casos también del virus de Epstein-Barr (VEB) $(11,12)$, aunque se desconoce la relación entre la infección por dichos virus y el desarrollo o promoción del crecimiento tumoral. Sin embargo, la escasa disponibilidad en muchos centros de estas pruebas tan específicas, junto con las peculiaridades clínicas y citológicas de esta entidad, hacen que no sean imprescindibles estas determinaciones para establecer su diagnóstico. Por otra parte, aunque algunos autores apuntan que es más frecuente la aparición de LPC en pacientes seropositivos para VIH que tengan historia previa de sarcoma de Kaposi (13), en nuestra causística el antecedente clínico de

\section{TABLA I}

\begin{tabular}{|c|c|c|c|}
\hline & Paciente 1 & Paciente 2 & Paciente 3 \\
\hline Edad/Sexo & 39/varón & 32/varón & 40/varón \\
\hline Epidemiología & ADVP & Homosexual & Homosexual \\
\hline Estadio Infección & $\mathrm{C} 3$ & $\mathrm{C3}$ & B3 \\
\hline Otras infecciones & $\begin{array}{l}\mathrm{VHC}+, \mathrm{HbcAc}+ \\
\text { TBC activa }\end{array}$ & No & No \\
\hline Linfocitos CD4 + & $20 / \mathrm{mm}^{3}$ & $128 / \mathrm{mm}^{3}$ & $28 / \mathrm{mm}^{3}$ \\
\hline Carga viral & Indetectable & 631 copias $/ \mathrm{mL}$ & 177.000 copias/mL \\
\hline Características del Líquido pleural & $\begin{array}{l}\text { Leu } 2,5 \times 10^{9} / \mathrm{L} \\
\text { Predominio linfocitario (70\%) } \\
80 \% \text { blastos } \\
\text { Proteínas } 4,6 \mathrm{~g} / \mathrm{dL}\end{array}$ & $\begin{array}{l}\text { Leu } 3,5 \times 10^{9} / \mathrm{L} \\
\text { Predominio linfocitario(80\%) } \\
52 \% \text { blastos } \\
\text { Proteínas } 5,6 \mathrm{~g} / \mathrm{dL}\end{array}$ & $\begin{array}{l}\text { Leu } 6,6 \times 10^{9} / \mathrm{L} \\
\text { Predominio linfocitario (85\%) } \\
50 \% \text { blastos } \\
\text { Proteínas } 5 \mathrm{~g} / \mathrm{dL}\end{array}$ \\
\hline LDH suero & $848 \mathrm{U} / \mathrm{L}$ & Normal & $933 \mathrm{U} / \mathrm{L}$ \\
\hline LDH líq. Pleural & $868 \mathrm{U} / \mathrm{L}$ & $9567 \mathrm{U} / \mathrm{L}$ & $968 \mathrm{U} / \mathrm{L}$ \\
\hline $\begin{array}{l}\text { Inmunofenotipo (positividad si } \\
\text { células que expresan el } \\
\text { AcMo es }>20 \% \text { ) }\end{array}$ & $\begin{array}{l}\text { Positivos: CD45, CD71, } \\
\text { HLA-Dr } \\
\text { Negativos: CD3, CD19, CD10, } \\
\text { CD79b, CD34, CD38, CD14 } \\
\text { CD64 }\end{array}$ & $\begin{array}{l}\text { Positivos: CD45, CD71, } \\
\text { HLA-Rr, CD79b, CD34, CD38 } \\
\text { Negativos: CD3, CD19, CD14, } \\
\text { CD64 }\end{array}$ & $\begin{array}{l}\text { Positivos: CD45, CD71, } \\
\text { HLA-Dr, CD79b, CD34, CD38 } \\
\text { Negativos: CD3, CD19, CD14, } \\
\text { CD64 }\end{array}$ \\
\hline Historia de SK previo & No & Sí (10 meses antes) & No \\
\hline Adenopatías & Sí (tuberculosas) & Sí (Kaposi) & No \\
\hline Esplenomegalia & Sí (moderada) & No & No \\
\hline Biopsia medular & Afectada & Inespecífica & Inespecífica \\
\hline L. Cefalorraquídeo & No afecto & No afecto & No afecto \\
\hline Tratamiento & CHOP al $75 \%$ & CHOP al $75 \%$ & CHOP al $75 \%$ \\
\hline Evolución & Éxitus en 24 horas & Éxitus en 24 meses & Vivo a los 4 meses. \\
\hline Tiempo VIH-LPC & 8 años & 6 meses & Simultáneo \\
\hline
\end{tabular}


sarcoma de Kaposi no siempre estuvo presente, confirmándose sólo en uno de los tres casos de LPC.

El diagnóstico de LPC obliga a descartar afectación ganglionar linfomatosa, es decir, debemos biopsiar las adenopatías que puedan encontrarse de forma concomitante en el momento de detectar el derrame específico, para comprobar que no sean de origen linfomatoso; si lo fueran, la afectación serosa debe entenderse secundaria a un linfoma nodal primario. Esta biopsia de eventuales adenopatías puede poner de manifiesto un origen tuberculoso de las mismas, o la coexistencia de enfermedad de Kaposuxxi ganglionar, resultados que validarían el diagnóstico de LPC. Estas posibilidades se dieron en dos de los tres casos que referimos: uno de ellos fue diagnosticado de tuberculosis ganglionar y el otro de Kaposi ganglionar (Fig. 1).

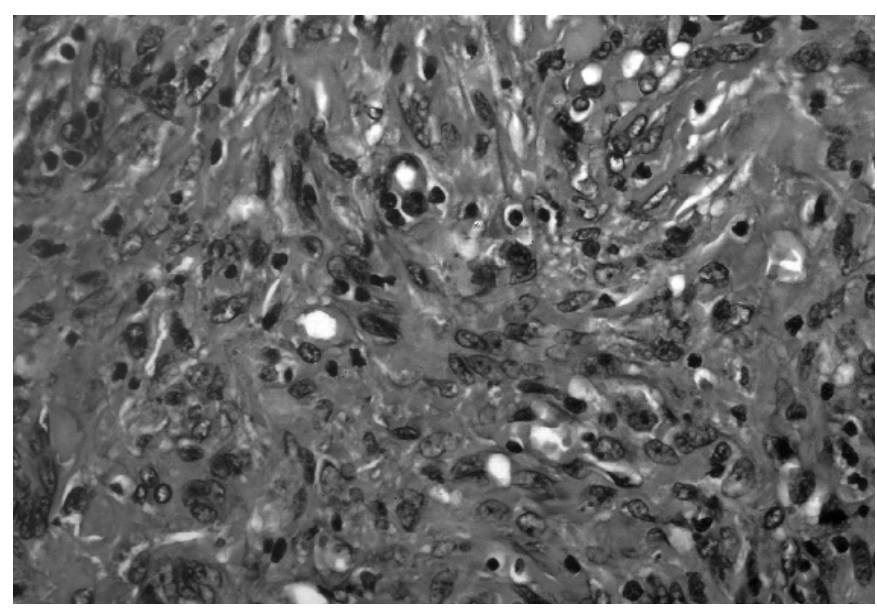

Fig. 1. Caso 1: Líquido pleural, May-Grünwald-Giemsa, x1000.

Desde un punto de visto citológico (Fig. 2), la morfología de la célula maligna plantea diagnóstico diferencial principalmente con un linfoma difuso de células grandes B (variante inmunoblástica) o con un linfoma anaplásico $(7,14)$, por lo que puede ser de gran ayuda realizar un análisis inmunofenotípico de una muestra celular del derrame. En este sentido, las primeras comunicaciones de este tipo de linfomas indicaban la ausencia de marcadores fenotípicos de línea B o T, pero sî la expresión de CD45 (antígeno panleucocitario común) y de marcadores para antígenos de activación (CD30, CD38, CD71 y HLA-DR); es por ello que inicialmente se pensaba en una naturaleza linfoide no-B no-T para este tipo de neoplasias. Sin embargo, la introducción de nuevos marcadores monoclonales y el estudio por PCR de reordenamientos propios de línea, han puesto de manifiesto que se trata en la inmensa mayoría de casos de una neoplasia linfoide B; con todo, se ha comunicado de forma aislada algún caso con fenotipo linfoide T (15). También se ha comunicado la expresión de CD138 (syndecan-1) en este tipo de neoplasias (16), marcador descrito como bastante específico para células plasmáticas. En nuestros pacientes, las células tumorales analizadas mediante citometría de flujo, fueron siempre positivas para CD45, CD71 y HLA-DR, y en dos casos también para CD79b (marcador específico de línea B), CD34 y CD38.

Aunque presenta poca tendencia a la diseminación, este tipo de linfoma puede mostrarse agresivo localmente, con fenómenos de destrucción tisular. Además, la respuesta al tratamiento es por

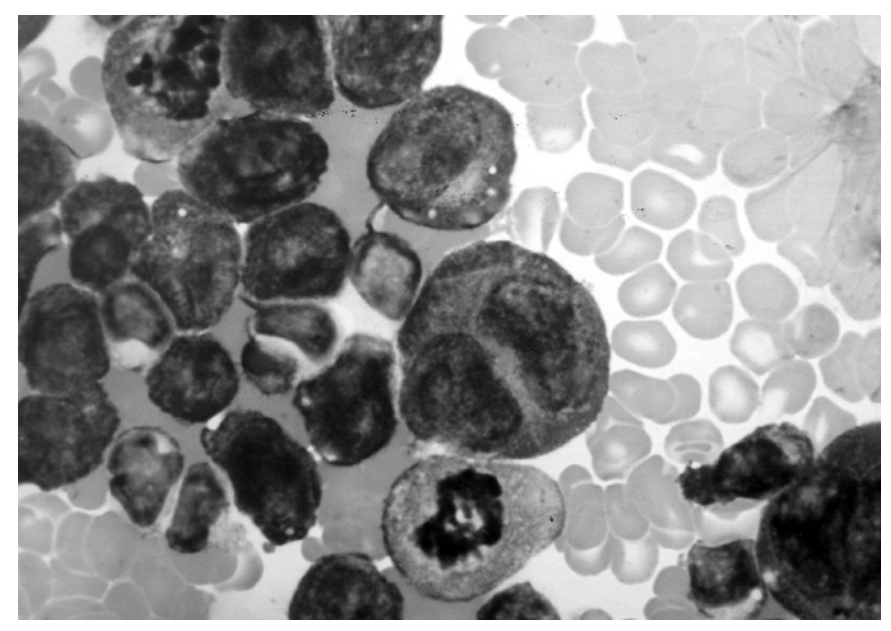

Fig. 2. Caso 2: Biopsia ganglionar (Kaposi ganglionar), Hematoxilina-eosina, $\times 400$.

lo general decepcionante, todo lo cual hace que tenga un pronóstico muy desfavorable, con una supervivencia media inferior a seis meses. El tratamiento por este motivo no está bien definido, habiéndose ensayado diversos esquemas poliquimoterápicos usado en otros linfomas de alto grado, siendo el más utilizado el esquema CHOP modificado (ciclofosfamida $600 \mathrm{mg} / \mathrm{m}^{2}$ x 1 día; doxorrubicina $45 \mathrm{mg} / \mathrm{m}^{2}$ x 1 día; vincristina $1,4 \mathrm{mg} / \mathrm{m}^{2}$, hasta un máximo de 2,0 mg, x 1 día; y prednisona $60 \mathrm{mg} /$ día x 5 días), administrándose un ciclo cada 28 días. Tras dos ciclos se reevalúa la respuesta (determinada a partir de la reducción del volumen del derrame). Si no hay respuesta, es improbable que ciclos adicionales sean efectivos; por el contrario, si existe respuesta, se administran otros 4-6 ciclos. Otros regímenes de quimioterapia usados más recientemente incluyen la doxorrubicina o daunorrubicina liposomal pegiladas; cuando ésta última ha sido administrada a dosis de $100 \mathrm{mg} / \mathrm{m}^{2}$ ha tenido al menos un efecto paliativo, incluso en aquellos casos en los que no se ha obtenido beneficio con el esquema CHOP mencionado.

Como en todos los linfomas asociados al sida, es muy importante administrar tratamiento antirretroviral altamente activo, pues la recuperación del sistema inmune, cuando sea posible, puede tener un gran efecto antitumoral adyuvante. Por otra parte, ocasionalmente se ha utilizado la radioterapia local en la cavidad afecta, con la que se ha logrado un efecto paliativo durante unos 12 meses aproximadamente.

Estudios preliminares han evidenciado que la actividad NF-B es necesaria para la supervivencia de células linfomatosas infectadas por el KSHV (17); la inhibición farmacológica de este factor de transcripción podría constituir en el futuro un tratamiento efectivo para este tipo de neoplasias. Así mismo, las células del LPC expresan en membrana el receptor de la interleukina-6 (IL-6), cuya estimulación por esta citoquina favorece el crecimiento del tumor (18). Oligonucleótidos IL-6 antisentido podrían utilizarse desde un punto de vista terapéutico para intentar controlar la clona neoplásica. Finalmente, en un trabajo reciente se ha comunicado una respuesta clínica significativa en un paciente con la utilización conjunta de azidotimidina (AZT) e interferón alfa, mediada al parecer por la inhibición que ejercen estos fármacos sobre la actividad NFkappaB mencionada anteriormente, a la vez que la activación del ligando inductor de la apoptosis relacionada con el factor de necrosis tumoral (TRAIL) (19). 


\section{Bibliografía}

1. Dal Maso L, Franceschi S. Epidemiology of non-Hodgkin lymphomas and other haemolymphopoietic neoplasms in people with AIDS. Lancet Oncol 2003; 4: 110-9.

2. Morassut S, Vaccher E, Balestreri L, Gloghini A, Gaidano G, Volpe R, et al. HIV-associated human herpesvirus 8-positive primary lymphomatous effusions: radiologic findings in six patients. Radiology 1997; 203: 459-63.

3. Banks PM, Warnke RA. Primary effusion lymphoma. En: Jaffe ES, Harris NL, Stein H, Vardiman JW, eds. Tumours of haematopoietic and lymphoid tissues. World Health Organization Classification of Tumours. Lyon, IARC Press; 2001, p. 179-80.

4. Jones D, Ballestas ME, Kaye KM, Gulizia JM, Winters GL, Fletcher J, et al. Primary-effusion lymphoma and Kaposi's sarcoma in a cardiactransplant recipient. N Engl J Med 1998; 339: 444-9.

5. Dotti G, Fiocchi R, Motta T, Facchinetti B, Chiodini B, Borleri GM, et al. Primary effusion lymphoma after heart transplantation: a new entity associated with human herpesvirus-8. Leukemia 1999; 13: 664-70.

6. Said JW, Tasaka T, Takeuchi S, Asou H, de Vos S, Cesarman E, et al Primary effusion lymphoma in women: report of two cases of Kaposi's sarcoma herpes virus-associated effusion-based lymphoma in human immunodeficiency virus-negative women. Blood 1996; 88: 3124-8.

7. Nador RG, Cesarman E, Chadburn A, Dawson DB, Ansari MO, Sald J, et al. Primary effusion lymphoma: A distinct clinico-pathologic entity associated with the Kaposi's sarcoma-associated herpes virus. Blood 1996; 88: 645-56.

8. Cesarman E, Chang Y, Moore PS, Said JW, Knowles DM. Kaposi's sarcoma-associated herpesvirus-like DNA sequences in AIDS related body-cavity-based lymphomas. N Engl J Med 1995; 332:1186-91.

9. Karcher DS, Alkan S. Human herpesvirus-8-associated body cavitybased lymphoma in human immunodeficiency virus-infected patients: a unique B-cell neoplasm. Hum Pathol 1997; 28: 8018.

10. Cannon M, Philpott NJ, Cesarman E. The Kaposi's sarcoma-associated herpesvirus $\mathrm{G}$ proteín-coupled receptor has broad signaling effects in primary effusion lymphoma cells. J Virol 2003; 77: 57-67.
11. Horenstein MG, Nador RG, Chadburn A, Hyjek EM, Inghirami G, Knowles DM, et al. Epstein-Barr virus latent gene expression in primary effusion lymphomas containing Kaposi's sarcoma-associated herpesvirus/human herpesvirus-8. Blood 1997; 90: 1186-91.

12. Komanduri KV, Luce JA, McGrath MS, Herndier BG, Ng VL. The natural history and molecular heterogeneity of HIV-associated primary malignant lymphomatous effusions. J Acquir Immune Defic Syndr Hum Retrovirol 1996; 13: 215-26.

13. Ansari MQ, Dawson DB, Nador R, Rutherford C, Schneider NR, Latimer MJ, et al. Primary body cavity-based AIDS-related lymphomas. Am J Clin Pathol 1996; 105: 221-9.

14. Ascoli V, Mastroianni CM, Galati V, Sirianni MC, Fruscalzo A, Pistilli A, et al. Primary effusion lymphoma containing human herpesvirus 8 DNA in two AIDS patients with Kaposi's sarcoma. Haematologica 1998; 83: 8-12.

15. Said JW, Shintaku IP, Asou H, de Vos S, Baker J, Hanson G, et al. Herpesvirus 8 inclusions in primary effusion lymphomas: report of a unique case with T-cell phenotype. Arch Pathol Lab Med 1999; 123: 257-60.

16. Gaidano G, Gloghini A, Gattei V, Rossi MF, Cilia AM, Godeas C, et al. Association of Kaposi's sarcoma-associated herpesvirus-positive primary effusion lymphoma with expression of the CD138/syndecan-1 antigen. Blood 1997; 90: 4894-900.

17. Keller SA, Schattner EJ, Cesarman E. Inhibition of NF-kappaB induces apoptosis of KSHV-infected primary effusion lymphoma cells. Blood 2000; 96: 2537-42.

18. Asou H, Said JW, Yang R, Munker R, Park DJ, Kamada N, et al. Mechanisms of growth control of Kaposi's sarcoma-associated herpes virus-associated primary effusion lymphoma cells. Blood 1998; 91: 2475-81.

19. Ghosh SK, Wood C, Boise LH, Mian AM, Deyev VV, Feuer G, et al. Potentiation of TRAIL-induced apoptosis in primary effusion lymphoma through azidothymidine-mediated inhibition of NF-kappaB. Blood $2003 ; 2321-7$. 